\title{
Quantitative prediction of effective toughness at random heterogeneous interfaces
}

\author{
Sylvain Patinet, ${ }^{1,2}$ Damien Vandembroucq, ${ }^{1,3}$ and Stéphane Roux ${ }^{4}$ \\ ${ }^{1}$ Laboratoire PMMH, ESPCI/CNRS-UMR 7636/Univ. Paris 6 UPMC/Univ. Paris 7 Diderot \\ 10 rue Vauquelin, 75231 Paris cedex 05, France \\ ${ }^{2}$ Dep. of Materials Science and Engineering, Johns Hopkins University, Baltimore, Maryland 21218, USA \\ ${ }^{3}$ The Niels Bohr Institut, Blegdamsvej 17, DK-2100 Copenhagen, Denmark \\ ${ }^{4}$ LMT-Cachan, ENS de Cachan/CNRS-UMR 8535/Univ. Paris 6 UPMC/PRES UniverSud Paris \\ 61 Avenue du Président Wilson, 94235 Cachan cedex, France
}

(Dated: May 31, 2022)

\begin{abstract}
The propagation of an adhesive crack through an anisotropic heterogeneous interface is considered. Tuning the local toughness distribution function and spatial correlation is numerically shown to induce a transition between weak to strong pinning conditions. While the macroscopic effective toughness is given by the mean local toughness in case of weak pinning, a systematic toughness enhancement is observed for strong pinning (the critical point of the depinning transition). A selfconsistent approximation is shown to account very accurately for this evolution, without any free parameter.
\end{abstract}

Introduction - While physicists studied the scaling properties of crack[1, 2] and developed an analogy between crack front propagation and the dynamical phase transition associated with the pinning/depinning of an elastic line driven through a random potential [3 12], a parallel (and independent) effort was made by mechanical engineers studying crack trapping by tough particles 13 15] or the effect of crack front deflection on the stress intensity factors (see e.g. [16] for a recent review).

Although the intimate link between surface energy of a material, and the resisting "force", or toughness, opposing interfacial crack propagation has been elucidated in the ideal cleavage case, the same concept remains to be better understood in more common situations where solids are heterogeneous. Generally, dissipative processes in the bulk of the solid (yet in the vicinity of the crack surface) contribute to (or even dominates over) the thermodynamic surface energy. This effect has been highlighted in recent studies [17, 18] showing how periodic modulations of elastic or interface properties affect crack propagation and considerably enhance the effective toughness of a given interface. Until recently 19 21], however, the computation of such an effective macroscopic toughness for random media has remained mostly unexplored despite its great theoretical (critical point of the depinning transition) and practical importance (optimized bonding).

When a crack propagates in a random solid heterogeneities may also trigger different dissipative phenomena, resulting in a toughness which cannot be reduced to the bare surface energy. Depending on the relative strength of the random potential and elasticity of the crack front, one usually distinguishes two generic situations 22] (see Fig. 1):

- In weak disorder conditions, the depinning front is only slightly perturbed and smoothly advances as a whole, with modest velocity fluctuations. Viscous dissipation can indeed be turned to arbitrary low values in quasi- static conditions, and hence only the bare average surface energy will be relevant for the macroscopic toughness. Disorder plays only a very minor role (e.g. for the geometry of the crack front).

- In strong pinning conditions, the front advances intermittently, by series of localized micro-instabilities, the front roughness exhibits a non trivial scale free behavior. In contrast with the previous case, the local motion during a micro instability is no longer under the control of the experimentalist. The unbalance of elastic forces is compensated by local viscous friction until a new equilibrium configuration is reached. The external driving force does not interfere much with this local resolution of the disequilibrium. Yet, at a macroscopic scale, the accumulation of these micro-instabilities will contribute to a total energy dissipation that dresses the surface energy. A similar mechanism has long been proposed for solid friction [23 25], or plasticity [26, 27].

It is therefore crucial to quantify the onset of strong pinning and to evaluate effective toughness in a quantitative fashion. Such is the purpose of the present study. First a numerical model is introduced to account for the crack front of an adhesive crack propagating in a random field of local toughness. This anisotropy-induced weak-to-strong pinning transition is shown to severely affect the value of the depinning threshold, or macroscopic toughness. Our results are shown to confirm early prediction about the effect of anisotropy of toughness [19, 20]. The velocity fluctuations along the front, through the participation ratio computation, are also shown to characterize the weak or strong pinning regimes.

Numerical model - We consider here an interfacial crack front propagating in the $(z, x)$ plane in $x$-direction. The location of the crack front at time $t$ is denoted $h(z, t)$. In the framework of brittle fracture, propagation is ruled locally by the Griffith criterion that compares a driving force, the Stress Intensity Factor (SIF) $K$, that depends on the geometry and the external loading with a 


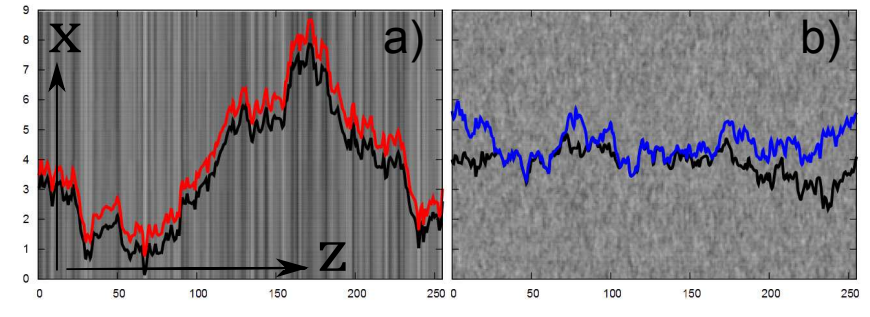

FIG. 1. Snapshots of crack fronts for different toughness landscapes illustrating the transition from weak (a) to strong (b) pinning regimes - same distribution $(\sigma=0.125)$, different correlation lengths: $\xi_{x}=50(\mathrm{a}), \xi_{x}=0.1$ (b). Two fronts separated by a small time lag are represented. In weak pinning conditions, the distance between successive fronts is nearly constant, indicating weak fluctuations of the local velocity. In strong pinning conditions, motion is concentrated over small parts of the front, indicating a jerky dynamics.

threshold value $K_{c}$, the toughness, a material property. The heterogeneity of the microscopic toughness is represented by a random landscape $K_{c}(z, x)$ of mean $\overline{K_{c}}$ and standard deviation $S_{c}$. It is assumed to be short-range correlated and its correlation lengths are denoted $\xi_{x}$ and $\xi_{z}$ in the direction of propagation and orthogonal to it respectively.

The microscopic toughness disorder induces a roughening of the crack front, which in turn modifies the local value of the SIF along the front via a long-range elastic restoration force [13]. Neglecting inertial effects, we consider in the following an over-damped dynamics. The local (forward) velocity is here given by the positive part of the difference between the local values of the SIF and the microscopic toughness 12]: $\mu \partial_{t} h=\mathcal{R}\left(K-K_{c}\right)$ where $\mu$ stands for an effective viscosity and $\mathcal{R}$ denotes the positive part. We study the behavior of the crack front at the verge of propagation from above, i.e. at a vanishing velocity for $K \approx \overline{K_{c}}$. This justifies a first order perturbative expansion around $\overline{K_{c}}$. The equation of evolution of the crack front thus writes [7, 11, 13]:

$$
\partial_{t} h(z, t)=\mathcal{R}\left[k_{0}(t)+k_{e l}(z, h(z, t))-k_{c}(z, h(z, t))\right]
$$

Here $k_{c}=K_{c} / \overline{K_{c}}$ is the reduced microscopic toughness landscape of unit mean, of standard deviation $\sigma=S_{c} / \overline{K_{c}}$ and of correlation lengths $\xi_{x}$ and $\xi_{z}$.

The driving force $k_{0}(t)=K_{0}(t) / \overline{K_{c}}$ is the reduced average SIF along the crack front. To account for the stiffness of the system (specimen and loading device), the boundary condition is described by a slow and steady loading rate such that on average the crack front velocity is set to $v_{0}$. A stiffness $e$ is introduced such that $k_{0}(t)=e\left[v_{0} t-\bar{h}(t)\right]$ where $\bar{h}(t)$ is the average position of the front at time $t$ [28]. The expression of the spatial modulation of the reduced SIF $k_{e l}$ as a function of the crack front geometry has been obtained to first order in perturbation [29] and writes

$$
k_{e l}(z, h(z))=\frac{1}{2 \pi} f \frac{h\left(z^{\prime}\right)-h(z)}{\left(z-z^{\prime}\right)^{2}} d z^{\prime}
$$

where $f$ stands for the principal value of the integral.

Note that in Eq.(11) the time scale has been set so that the viscosity is scaled to unity. Two parameters thus remain that characterize the driving dynamics: the (reduced) stiffness $e$ and the (reduced) velocity $v_{0}$. In the following only the quasi-static limit $v_{0} \rightarrow 0^{+}$is considered.
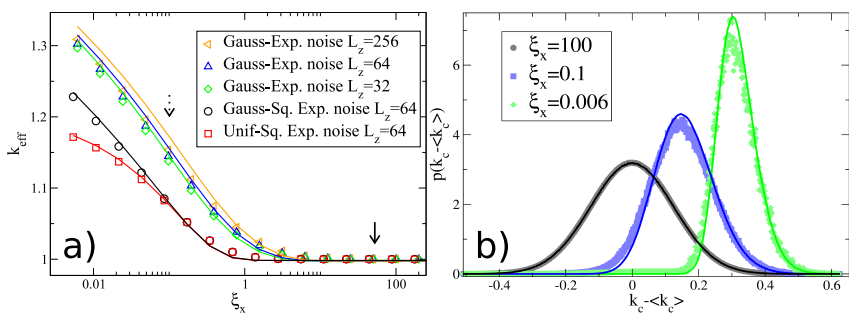

FIG. 2. (a) Effective toughness $k_{\text {eff }}$ (for $\sigma=0.125$ ) vs correlation length $\xi_{x}$ for different disorders types and system widths $L_{z}$. The continuous and dotted arrows indicate the weak and strong pinning regimes reported in Fig. 1/a and $1 \mathrm{p}$ respectively. (b) Toughness distribution along the crack front that propagates in an exponentially correlated Gaussian landscape of width $L_{z}=64$ for different $\xi_{x}(\sigma=0.125)$. Symbols indicate simulation data and lines theoretical predictions.

In the following, random toughness fields of size $L_{z} \times L_{x}$ are considered with a unit lateral correlation length $\xi_{z}=1$ (the discretization length scale in the lateral direction) and a tunable correlation length $\xi_{x}$ in the direction of propagation. Three different types of random fields were considered: Uniform-Squared Exponential (U-SE), Gaussian-Squared Exponential (G-SE) and GaussianExponential (G-E) where the first term refers to the probability distribution function of $k_{c}$ and the second one qualifies its autocorrelation function $C(\Delta x)$ in the $x$ direction. The U-SE and G-SE disorders consist of grids of $N_{z}\left(=L_{z} / \xi_{z}\right) \times N_{x}\left(=L_{x} / \xi_{x}\right)$ random numbers from uniform and Gaussian distributions respectively. The spacing between grids points in the $x$ direction follows a uniform distribution such that $C(\Delta x)=e^{-\left(\Delta x / \xi_{x}\right)^{2}}$. The G-E landscape consists of realizations of an exponentially correlated Gaussian noise $C(\Delta x)=e^{-\left(\Delta x / \xi_{x}\right)}$ computed according to first order scheme 30 with an integration step $\Delta x=\xi_{x} / 50$. In all cases, the continuous toughness landscape $k_{c}(z, x)$ is interpolated linearly between two grid points in the $x$ direction.

The standard deviation $\sigma$ is varied in the range [0.125$1]$ and the correlation length $\xi_{x}$ in the range [0.006 - 800] while the reduced stiffness is set to $e=1$. The chosen reduced velocity $v_{0}=\sigma / 20$ was verified to be small enough not to significantly influence the results. Periodic boundary conditions along $z$ are considered. Integration 
of Eq. 1] is performed according to an explicit mid-point scheme. The time step $\delta t$ is chosen so that the maximum front increment is less than on tenth of the noise discretization length. Starting from a flat configuration, the crack front was first propagated over $\xi_{x}\left(L_{z} / \xi_{z}\right)^{0.5}$ in order to reach a statistical steady state.

In the spirit of a homogenization approach, the effective toughness is defined as the one which would be measured at a macroscopic scale. The (reduced) effective toughness $k_{\text {eff }}$ is thus measured as the time (and ensemble) average of the (reduced) Stress Intensity Factor $k_{0}(t)$ along propagation for a vanishing velocity: $k_{\text {eff }}=\left\langle k_{0}(t)\right\rangle$. In practice $k_{\text {eff }}$ is computed as the mean value of the driving force minus the driving velocity $k_{0}-v_{0}$ along a propagation length equal to $L_{x}=1024 \xi_{x}$. This value is finally averaged again over ten simulations (different statistical samples).

Numerical results - The weak-to-strong pinning transition induced by the shortening of the toughness correlation length goes together with a spectacular increase of the effective toughness (the depinning threshold). Fig. 2a shows the dependence of $k_{\text {eff }}$ on different types of disorder. For large values of the correlation length $\xi_{x}$ we obtain $k_{\text {eff }}=1$ i.e. the effective toughness equals the mean of the microscopic disorder $\left\langle k_{c}\right\rangle$. However for low values of $\xi_{x}, k_{\text {eff }}$ departs from $\left\langle k_{c}\right\rangle=1$ and reaches significantly higher values that clearly depend on the type of distribution and correlation of the microscopic disorder. Indeed the effective toughness is interpreted here as the threshold of a (dynamic) phase transition and as such is expected to depend on the microscopic details. We also note a slight but clear dependence on the system size: the larger the system, the larger $k_{\text {eff }}$. This enhancement is also reflected by changes in the toughness distribution (weighted by time) visited by the crack front as illustrated in Fig. 2. This distribution is clearly biased towards higher toughness values as $\xi_{x}$ decreases.

Interpretation: a self-consistent approach - This evolution can be rationalized in the framework of a selfconsistent approximation proposed in Ref. [19], and never checked quantitatively. The top panel of Fig. 3 shows a toughness landscape consisting of a unique band of fluctuating toughness $k_{c}(x)$ in an otherwise homogeneous medium of toughness $k_{0}$. Because of the elastic coupling, the crack front undergoes a deflection $\Delta h=\bar{h}-h(0)$ proportional to the toughness contrast $\Delta k=k_{c}-k_{0}$. The associated line stiffness $S=\Delta k / \Delta h$ can be analytically computed from Rice formula 19, 31] and is shown to scale as $S \propto 1 / \xi_{z} \log \left(L_{z} / \xi_{z}\right)$. An effective medium approximation, in the spirit of the self-consistent approximation, consists in evaluating $k_{\text {eff }}$ as equal to the value of $k_{0}$ such that the average deflection of the front taken over the ensemble of successive stable positions of the front is zero.

From weak to strong pinning - The simplicity of the one-dimensional picture of Fig. 3 allows one to define a

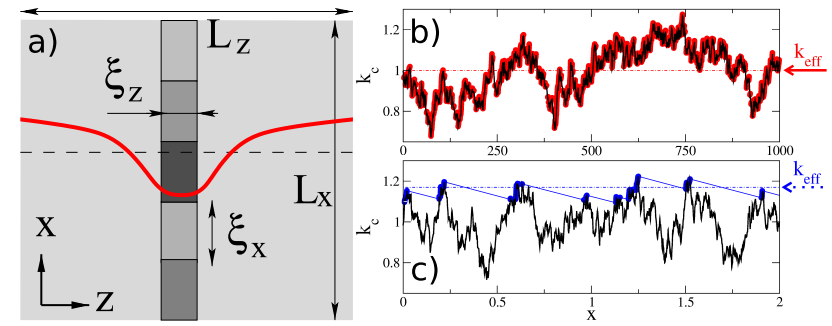

FIG. 3. (a) A band of random toughness in a homogeneous landscape traps the crack front; the lower the line stiffness, the larger the front deflection. (b) and (c) Graphical solutions of the implicit equation $\Delta k_{e l}(x)=\Delta k_{c}(x)$ which determines the equilibrium position of the crack front for the same disorder parameters reported in Fig. 19 and 10. The stable positions in the band are highlighted in red and blue respectively. For large correlation length (b), the trajectory almost follows the toughness in the band (weak pinning). For small correlation length (c), the equilibrium positions are skewed toward higher toughness (strong pinning). The slope of the fine lines between the stable portions corresponds to the stiffness $S$ of the crack front.

criterion that determines the pinning regime. In absence of driving force, an equilibrium configuration of the front at position $h(z=0, t)=x$ in the band is obtained when the elastic restoring force, $\Delta k_{e l}(x)=S \Delta h(x)$, balances the toughness contrast $\Delta k_{c}(x)$. Depending on the respective amplitude of the line stiffness and the local toughness gradient, one can obtain for this implicit equation either a unique solution or multiple solutions for the front position $x$. A simple criterion for the transition from weak to strong pinning can thus be drawn from the onset of multistability. Under these conditions, strong pinning is obtained when

$$
\frac{\sigma}{S \xi_{x}}>1
$$

As schematically shown in Fig. 3]b different sets of stable positions along the band are generated as a function of the line stiffness. For large $S \xi_{x} / \sigma$ the stable trajectory closely follows the toughness of the band as expected from the weak pinning regime. Conversely for lower $S \xi_{x} / \sigma$ the crack front will only visit a subset of high toughness values that characterize the strong pinning regime. As a consequence, the distribution of toughness at stable positions is skewed toward higher values.

Direct numerical integrations of the effective medium model have been performed. $S$ is first determined numerically to account for the discreteness of the simulated crack front. The reduced toughness distributions and their means are then generated from one dimensional trajectories as exemplified in Fig. 3a and b. The comparison between the self-consistent approximation and the crack front simulations reported in Fig. 2 and in Fig. 4 shows a remarkable agreement. $k_{\text {eff }}$ is very accurately reproduced as a function of $\xi_{x}, L_{z}, \sigma$ and the different disorder types. Note that the model not only accounts for the effective 
toughness variations but also for the visited toughness distributions without any free parameter.

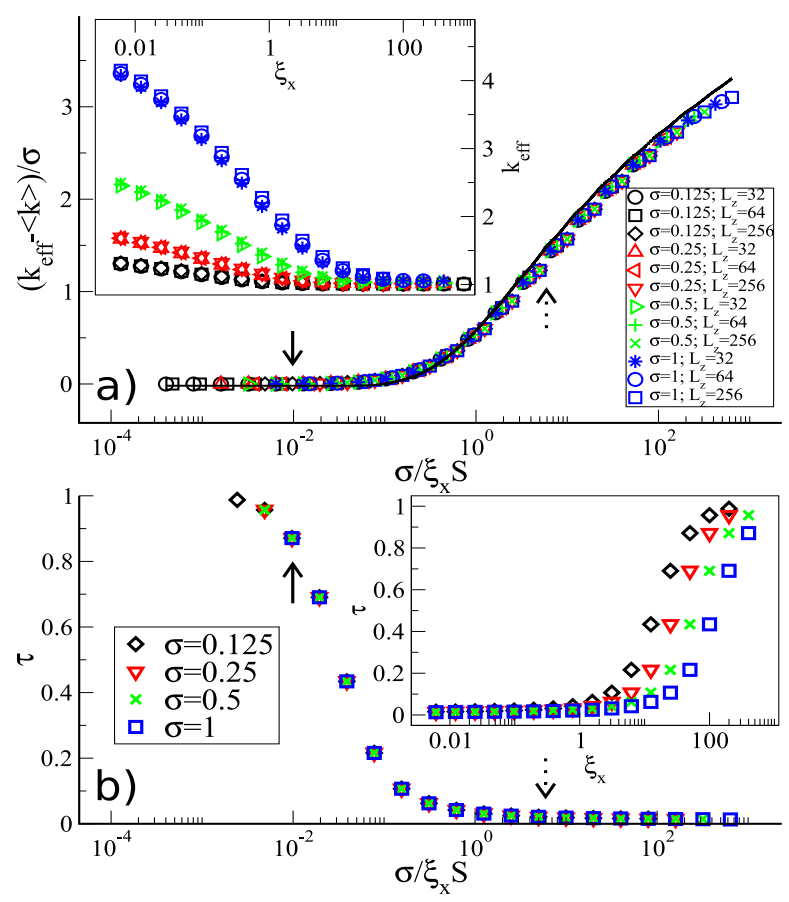

FIG. 4. (a) Effective toughness $k_{e f f}$ as a function of the correlation length $\xi_{x}$ for different landscape toughness standard deviations $\sigma$ and system widths $L_{z}$. The larger $\sigma$ and/or the shorter $\xi_{x}$, the larger $k_{\text {eff }}$. Symbols and lines correspond to simulation data and theoretical predictions respectively. (b) Participation ratio $\tau$ along a crack front of width $L_{z}=256$. The larger $\sigma$ and/or the shorter $\xi_{x}$, the lower $\tau$. In both figures, disorder type is GE. Original simulation data are reported in the insets while the main panels correspond to the rescaled quantities following Eq. 3 The continuous and dotted arrows indicate the weak and strong pinning regimes reported in Fig. 1ta and 10 respectively.

Scaling analysis - One can show that only a combination of scaled parameters really contributes to the observed results. If the toughness $k$, and distance $x$ along the propagation direction, are transformed into $k \rightarrow\left(k-\left\langle k_{c}\right\rangle\right) / \sigma$ and $x \rightarrow x / \xi_{x}$ then the elasticity of the crack front line becomes $S \xi_{x} / \sigma$. Fig. 媪 shows the centered and reduced effective toughness against the depinning control parameter $\sigma / S \xi_{x}$. All numerical results collapse onto a single master curve that captures the transition. For small values of the control parameter $\sigma / S \xi_{x}$ the weak pinning regime holds, $k_{\text {eff }}=1$, whereas, for large values $\sigma / S \xi_{x}, k_{\text {eff }}$ significantly increases (strong pinning). Note that the system size dependence is here captured in the line stiffness parameter which decreases as the logarithm of the crack front length. This has no effect in the weak-pinning regime, but justifies a rather counter-intuitive result in the framework of brittle fracture: the larger the system, the smaller the line stiffness and hence the larger the effective toughness.
Details of the local disorder have a dramatic effect on the pinning conditions. A crack front can encounter weak or strong pinning depending on its propagation direction. In the same spirit, if the toughness landscape is nonsymmetrical, the effective toughness in a given direction will not be the same as in the reverse one. Our approach thus offers a natural interpretation to the recent results presented in Ref. [18].

Crack front dynamics - As mentioned above, the weakto-strong pinning transition is also characterized by the emergence of intermittence and localization of the propagation. To quantify the localization degree along the front, the participation ratio [22] is computed

$$
\tau=\left\langle\frac{\left(\sum_{i=1}^{N_{z}} \delta h\left(z_{i}\right)^{2}\right)^{2}}{N_{z} \sum_{i=1}^{N_{z}} \delta h\left(z_{i}\right)^{4}}\right\rangle,
$$

where $\delta h\left(z_{i}\right)$ is the local velocity of the $i$ th site and $N_{z}$ the total number of site along the crack front. The brackets denote time average. This scalar parameter measures the relative number of sites involved during motion. In the weak pinning case where all sites move, $\tau=1$. In the strong pinning limit where only one site moves, $\tau=1 / N_{z}$ is expected. The numerical results reported in Fig. 4 $\mathrm{b}$ are fully consistent with this picture: the lower the correlation length $\xi_{x}$ and/or the larger the standard deviation $\sigma$ of the toughness disorder, the lower the participation ratio, i.e. the wider the velocity distribution. As shown in Fig. 48 the participation ratio data collapses onto a master curve describing the transition from weak to strong pinning for different disorder strengths and correlation lengths, validating again the relevance of the scaling parameter.

Conclusion - The depinning of a crack front has been shown to be strongly dependent on the spatial correlation of the disordered landscape through which it propagates. The transition between weak and strong pinning is well captured by a simple criterion built on the toughness gradient in the direction of propagation and the line stiffness. A simple self-consistent approximation very accurately describes the progressive departure of the depinning threshold from its weak pinning value (the mean value of the disorder) to the higher values measured for strong pinning. This scheme accurately captures the dependence of the depinning threshold to the finer microscopic details (statistical distribution of toughness and spatial correlation function). Let us emphasize that such a result may not have been expected from the fact that strong pinning involves micro-instabilities and collective phenomena (avalanches, ...) due to the underlying dynamical phase transition.

Bearing in mind that the enhancement of the effective toughness does not originate from the initial landscape toughness distribution only but also from its spatial correlation, our results open a promising route to design anisotropic and tough interfaces. 
S.P. acknowledges the support of ANR project MeßhyStaR.

E Bouchaud I Phys Cond Mat 9 4319 (1997)

[2] M. J. Alava, P. Nukala, and S. Zapperi, Adv. Phys., 55, 349 (2006).

[3] J. Schmittbuhl, S. Roux, J. P. Vilotte, and K. J. Måløy, Phys. Rev. Lett., 74, 1787 (1995).

[4] P. Daguier, B. Nghiêm, E. Bouchaud, and F. Creuzet, Phys. Rev. Lett., 78, 1062 (1997).

[5] S. Ramanathan and D. S. Fisher, Phys. Rev. Lett., 79, 877 (1997).

[6] S. Ramanathan, D. Ertaş, and D. S. Fisher, Phys. Rev. Lett., 79, 873 (1997).

[7] S. Ramanathan and D. S. Fisher, Phys. Rev. B, 58, 6026 (1998).

[8] A. Tanguy, M. Gounelle, and S. Roux, Phys. Rev. E, 58, 1577 (1998).

[9] S. Santucci, K. J. Måløy, A. Delaplace, J. Mathiesen, A. Hansen, J. O. Haavig Bakke, J. Schmittbuhl, L. Vanel, and P. Ray, Phys. Rev. E, 75, 016104 (2007).

[10] D. Dalmas, A. Lelarge, and D. Vandembroucq, Phys. Her. Ett., 101, 255501 (2008).

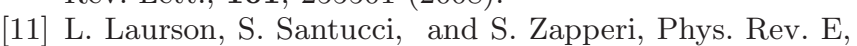
81, 046116 (2010).

[12] D. Bonamy and E. Bouchaud, Phys. Rep., 498, 1 (2011).

[13] H. J. Gao and J. R. Rice, J. Appl. Mech., 56, 828 (1989).
[14] A. F. Bower and M. Ortiz, J. Mech. and Phys. Solids, 39, 815 (1991).

[15] T. M. Mower and A. S. Argon, Mechanics of Materials, 19, 343 (1995).

[16] V. Lazarus, J. Mech. Phys. Solids, 59, 121 (2011).

[17] V. Tvergaard and J. W. Hutchinson, Int. J. Solids Struct., 46, 3433 (2009), ISSN 0020-7683.

[18] S. Xia, L. Ponson, G. Ravichandran, and K. Bhattacharya, Phys. Rev. Lett., 108, 196101 (2012).

[19] S. Roux, D. Vandembroucq, and F. Hild, Eur. J. Mech. A, 22, 743 (2003).

[20] S. Roux and F. Hild, Int. J. Fract., 154, 159 (2008).

[21] V. Démery, L. Ponson, and A. Rosso, arXiv:1212.1551 (2012).

[22] A. Tanguy and T. Vettorel, Eur. J. Phys. B, 38, 71 (2004).

[23] F. Heslot, T. Baumberger, B. Perrin, B. Caroli, and C. Caroli, Phys. Rev. E, 49, 4973 (1994).

[24] C. Caroli and P. Nozières, Eur. Phys. J. B, 4, 233 (1998).

[25] T. Baumberger and C. Caroli, Adv. Phys., 55, 279 (2006).

[26] G. Puglisi and L. Truskinovsky, J. Mech. Phys. Solids, 53, 655 (2005).

[27] A. Mielke and L. Truskinovsky, Arch. Rational Mech. Anal., 203, 577 (2012).

[28] D. Bonamy, S. Santucci, and L. Ponson, Phys. Rev. Lett., 101, 045501 (2008).

[29] J. R. Rice, J. Appl. Mech., 52, 571 (1985).

[30] R. F. Fox, I. R. Gatland, R. Roy, and G. Vemuri, Phys. Rev. A, 38, 5938 (1988).

[31] J. Chopin, A. Prevost, A. Boudaoud, and M. AddaBedia, Phys. Rev. Lett., 107, 144301 (2011). 